\title{
A note on the $\Theta$-invariant of 3-manifolds
}

\author{
Cattaneo, Alberto S ; Shimizu, Tatsuro
}

\begin{abstract}
In this note, we revisit the $\Theta$-invariant as defined by R. Bott and the first author in [4]. The $\Theta$-invariant is an invariant of rational homology 3 -spheres with acyclic orthogonal local systems, which is a generalization of the 2-loop term of the Chern-Simons perturbation theory. The $\Theta$-invariant can be defined when a cohomology group is vanishing. In this note, we give a slightly modified version of the $\Theta$-invariant that we can define even if the cohomology group is not vanishing.
\end{abstract}

DOI: https://doi.org/10.4171/qt/146

Posted at the Zurich Open Repository and Archive, University of Zurich ZORA URL: https://doi.org/10.5167/uzh-202230

Journal Article

Published Version

Originally published at:

Cattaneo, Alberto S; Shimizu, Tatsuro (2021). A note on the $\Theta$-invariant of 3-manifolds. Quantum Topology, 12(1):111-127.

DOI: https://doi.org/10.4171/qt/146 


\title{
A note on the $\Theta$-invariant of 3-manifolds
}

\author{
Alberto S. Cattaneo and Tatsuro Shimizu
}

\begin{abstract}
In this note, we revisit the $\Theta$-invariant as defined by R. Bott and the first author in [4]. The $\Theta$-invariant is an invariant of rational homology 3 -spheres with acyclic orthogonal local systems, which is a generalization of the 2-loop term of the ChernSimons perturbation theory. The $\Theta$-invariant can be defined when a cohomology group is vanishing. In this note, we give a slightly modified version of the $\Theta$-invariant that we can define even if the cohomology group is not vanishing.
\end{abstract}

Mathematics Subject Classification (2020). 57R56, 81Q30.

Keywords. Invariants of 3-manifolds, Chern-Simons perturbation theory, configuration space integral.

\section{Introduction}

In 1998, R. Bott and the first author defined topological invariants of rational homology spheres with acyclic orthogonal local systems in [3] and [4]. These invariants were inspired by the Chern-Simons perturbation theory developed by M. Kontsevich in [6], S. Axelrod and M. I. Singer in [2]. The Chern-Simons perturbation theory gives invariants of 3-manifolds with flat connections of the trivial $G$-bundle over the 3-manifold, where $G$ is a semi-simple Lie group. The composition of adjoint representation of $G$ and the holonomy representation of the flat connection gives an orthogonal local system.

In [4], Bott and the first author constructed a real valued invariant, called $\Theta$-invariant (In this note, we denote by $Z_{\Theta}$ the corresponding term), which is a generalization of a 2-loop term of Chern-Simons perturbation theory. The vanishing of a cohomology group (denoted by $H_{-}^{*}\left(\Delta ; \pi_{1}^{-1} E \otimes \pi_{2}^{-1} E\right)$ in [4], $H_{-}^{*}\left(\Delta ; E_{\rho} \otimes E_{\rho}\right)$ in this note) plays an important role in the construction of the $\Theta$-invariant $Z_{\Theta}$. There are few gaps in the proof of this vanishing (Lemma 1.2 of [4]). In this note, we show that a linear combination of $Z_{\Theta}$ and another term $Z_{O-O}$ is, however, a topological invariant of closed 3-manifolds with orthogonal acyclic local systems, 
when the local system is given by using a holonomy representation of a flat connection. The term $Z_{O-O}$ is also related to the 2-loop term of the Chern-Simons perturbation theory. We note that the second author proved that when $G=\mathrm{SU}(2)$, $Z_{\Theta}$ itself is an invariant of closed 3-manifolds with orthogonal local systems in [9].

The organization of this paper is as follows. In Section 2 we give a modified version of the Bott-Cattaneo $\Theta$-invariant without proof. In Section 3 and Section 4 we prove a proposition and a theorem about consistency of the definition of Section 2. Both the invariant defined in Section 2 of this note and the $\Theta$-invariant depend on the choice of a framing of the 3-manifold. In Section 5 we introduce a framing correction.

Orientation convention. In this note, all manifolds are oriented. Boundaries are oriented by the outward normal first convention. Products of oriented manifolds are oriented by the order of the factors. The interval $[0,1] \subset \mathbb{R}$ is oriented from 0 to 1 .

Acknowledgments. Alberto S. Cattaneo acknowledges partial support of SNF Grant No. 200020 172498/1. This research was (partly) supported by the NCCR SwissMAP, funded by the Swiss National Science Foundation, and by the COST Action MP1405 QSPACE, supported by COST (European Cooperation in Science and Technology). Tatsuro Shimizu expresses his appreciation to Professor Tadayuki Watanabe for his helpful comments and discussion on the Chern-Simons perturbation theory. This work was (partly) supported by JSPS KAKENHI Grant Number JP15K13437.

\section{The invariant}

Let $M$ be a closed oriented framed 3-manifold, namely a trivialization of the tangent bundle of $M$ is fixed. We take a metric on $M$ compatible with the framing. Let $\rho: \pi_{1} \rightarrow G$ be a representation of the fundamental group into a semi-simple Lie group $G$. We denote by $\operatorname{Ad}: G \rightarrow \operatorname{Aut}(\mathfrak{g})$ the adjoint representation of $G$, where $\mathfrak{g}$ is the Lie algebra of $G$. Since $G$ is semi-simple, the Killing form of $\mathfrak{g}$ is non-degenerate. Since $\operatorname{Ad}(g)$ preserves the Killing form for any $g \in \mathfrak{g}$, the representation $\mathrm{Ad} \circ \rho$ is orthonormal with respect to the Killing form. A local system is a covariant functor from the fundamental groupoid of $M$ to the category of finite dimensional vector spaces. Note that a representation of $\pi_{1}(M)$ gives a local system. We denote by $E_{\rho}$ the local system given by $\operatorname{Ad} \circ \rho$. We assume that 
$E_{\rho}$ is acyclic, namely

$$
H^{*}\left(M ; E_{\rho}\right)=0 .
$$

In this note, we say that such a representation $\rho$ is acyclic.

2.1. A compactification of a configuration space. Let $\Delta=\{(x, x): x \in M\} \subset$ $M^{2}$ be the diagonal. We identify $\Delta$ with $M$ by

$$
\Delta \ni(x, x) \longrightarrow x \in M .
$$

We orient $\Delta$ by using this identification. We denote by $v_{\Delta}$ the normal bundle of $\Delta$ in $M^{2}$. We identify $v_{\Delta}$ with the tangent bundle $T M$ via the isomorphism defined by

$$
T M \stackrel{\cong}{\longrightarrow} v_{\Delta}, \quad(x, v) \longmapsto((x, x),(-v, v))
$$

where $x \in M$ and $v \in T_{x} M$. On the other hand, $M$ is framed. Then $T M$ is identified with $M \times \mathbb{R}^{3}$. Thus $v_{\Delta}$ is identified with $M \times \mathbb{R}^{3}$.

Let $C_{2}(M)=B \ell\left(M^{2}, \Delta\right)$ be the compact 6-dimensional manifold with the boundary obtained by the real blowing up of $M^{2}$ along $\Delta$. We denote by

$$
q: C_{2}(M) \longrightarrow M^{2}
$$

the blow-down map. As manifolds,

$$
C_{2}(M)=\left(M^{2} \backslash \Delta\right) \cup S v_{\Delta}
$$

and $q\left(S v_{\Delta}\right)=\Delta$. Here $S v_{\Delta}$ is the unit sphere bundle of $v_{\Delta}$ with respect to the metric on $M$. The manifold $C_{2}(M)$ is a compactification of the configuration space $M^{2} \backslash \Delta$ of two distinct points. Obviously, $\partial C_{2}(M)=S v_{\Delta}$.

$S v_{\Delta}$ is identified with $\Delta \times S^{2}$. We denote by

$$
p: \partial C_{2}(M)=\Delta \times S^{2} \longrightarrow S^{2}
$$

the projection. We use the same symbol $q$ for the restriction map

$$
\left.q\right|_{\partial C_{2}(M)}: \partial C_{2}(M)\left(=\Delta \times S^{2}\right) \longrightarrow \Delta
$$

of the blow-down map $q$.

2.2. The natural transformations $c$ and Tr. The Killing form gives an isomorphism $\mathfrak{g} \otimes \mathfrak{g} \cong \mathfrak{g}^{*} \otimes \mathfrak{g}^{*}$. Let $\mathbf{1} \in \mathfrak{g} \otimes \mathfrak{g}$ the element corresponding to the Killing form in $\mathfrak{g}^{*} \otimes \mathfrak{g}^{*}$. By using an orthonormal basis $e_{1}, \ldots, e_{\operatorname{dim} \mathfrak{g}} \in \mathfrak{g}$ of $\mathfrak{g}, \mathbf{1}$ can be described as

$$
\mathbf{1}=\sum_{i=1}^{\operatorname{dim} \mathfrak{g}} e_{i} \otimes e_{i} .
$$


$\mathbf{1} \in \mathfrak{g} \otimes \mathfrak{g}$ is invariant under the diagonal action of $\pi_{1}(M)$. Thus we have a natural transformation

$$
c: \underline{\mathbb{R}} \longrightarrow E_{\rho} \otimes E_{\rho}, \quad 1 \longmapsto \mathbf{1} .
$$

Here $\underline{\mathbb{R}}$ is the trivial local system, namely a local system corresponding to the 1-dimensional trivial representation of $\pi_{1}(M)$.

We define a natural transformation

$$
\operatorname{Tr}: E_{\rho} \otimes E_{\rho} \otimes E_{\rho} \longrightarrow \underline{\mathbb{R}}
$$

as follows: for $x, y, z \in \mathfrak{g}$,

$$
\operatorname{Tr}(x \otimes y \otimes z)=\langle[x, y], z\rangle
$$

where $\langle$,$\rangle is the Killing form and [, ] is the Lie bracket.$

Let $\pi_{1}, \pi_{2}: M^{2} \rightarrow M$ be the projections defined by

$$
\pi_{1}\left(x_{1}, x_{2}\right)=x_{1}, \quad \pi_{2}\left(x_{1}, x_{2}\right)=x_{2} .
$$

$\pi_{1}^{*} E_{\rho} \otimes \pi_{2}^{*} E_{\rho}$ is a local system on $M^{2}$. We denote

$$
E_{\rho} \otimes E_{\rho}=\pi_{1}^{*} E_{\rho} \otimes \pi_{2}^{*} E_{\rho} .
$$

We remark that $\left.E_{\rho} \otimes E_{\rho}\right|_{\Delta}=E_{\rho} \otimes E_{\rho}$. The pull-back

$$
F_{\rho}=q^{*}\left(E_{\rho} \otimes E_{\rho}\right)
$$

is a local system on $C_{2}(M)$. Clearly, $\left.F_{\rho}\right|_{\partial C_{2}(M)}=q^{*}\left(E_{\rho} \otimes E_{\rho}\right)$.

2.3. The involution $\boldsymbol{T}$ on $\boldsymbol{C}_{2}(\boldsymbol{M})$. The involution $T_{0}: M^{2} \rightarrow M^{2}$ defined by $T_{0}\left(x_{1}, x_{2}\right)=\left(x_{2}, x_{1}\right)$ induces an involution $T: C_{2}(M) \rightarrow C_{2}(M) . T_{0}, T$ induce homomorphisms $T_{0}^{*}, T^{*}$ on the cohomology groups $H^{*}\left(M^{2}, E_{\rho} \otimes E_{\rho}\right)$, $H^{*}\left(C_{2}(M) ; F_{\rho}\right)$, and $H^{*}\left(\Delta ; E_{\rho} \otimes E_{\rho}\right)$, and on the space of differential $k$-forms $\Omega^{k}\left(C_{2}(M) ; F_{\rho}\right)$. We denote by $H_{+}^{*}\left(M^{2} ; E_{\rho} \otimes E_{\rho}\right)$ and $H_{-}^{*}\left(M^{2} ; E_{\rho} \otimes E_{\rho}\right)$ the $+1,-1$ eigenspaces of the homomorphism $T_{0}^{*}$ respectively. We use similar notations $H_{+}^{*}\left(C_{2}(M) ; F_{\rho}\right), H_{+}^{*}\left(\Delta, E_{\rho} \otimes E_{\rho}\right), \Omega_{+}^{k}\left(C_{2}(M) ; F_{\rho}\right), \ldots$ in the same manner.

Let $T_{S^{2}}: S^{2} \rightarrow S^{2}$ be the involution defined as

$$
T_{S^{2}}(x)=-x \quad \text { for any } x \in S^{2} .
$$

We remark that $\left.p \circ T\right|_{\partial C_{2}(M)}=T_{S^{2}} \circ p: \partial C_{2}(M) \rightarrow S^{2}$. 
2.4. The invariant. Take a 2 -form $\omega_{S^{2}} \in \Omega^{2}\left(S^{2} ; \mathbb{R}\right)$ on $S^{2}$ satisfying

$$
\int_{S^{2}} \omega_{S^{2}}=1
$$

and

$$
T_{S^{2}}^{*} \omega_{S^{2}}=-\omega_{S^{2}} \text {. }
$$

The form $p^{*} \omega_{S^{2}}$ is a closed 2-form on $\partial C_{2}(M)$. Thus

$$
c_{*}\left(p^{*} \omega_{S^{2}}\right)=p^{*} \omega_{S^{2}} \mathbf{1}
$$

is a closed 2-form on $\partial C_{2}(M)$ such that $\left(\left.T\right|_{C_{2}(M)}\right)^{*} p^{*} \omega_{S^{2}} \mathbf{1}=-p^{*} \omega_{S^{2}} \mathbf{1}$. The closed 2-form $p^{*} \omega_{S^{2}} 1$ represents a cohomology class in $H_{-}^{2}\left(\partial C_{2}(M) ;\left.F_{\rho}\right|_{\partial C_{2}(M)}\right)$ :

$$
\left[p^{*} \omega_{S^{2}} \mathbf{1}\right] \in H_{-}^{2}\left(\partial C_{2}(M) ;\left.F_{\rho}\right|_{\partial C_{2}(M)}\right) .
$$

Proposition 2.1. There exist closed 2-forms

$$
\omega \in \Omega^{2}\left(C_{2}(M) ; F_{\rho}\right) \quad \text { and } \xi \in \Omega^{2}\left(\Delta ; E_{\rho} \otimes E_{\rho}\right)
$$

satisfying the following conditions:

(1) $\left.\omega\right|_{\partial C_{2}(M)}=p^{*} \omega_{S^{2}} \mathbf{1}+q^{*} \xi$,

(2) $T^{*} \omega=-\omega,\left(\left.T_{0}\right|_{\Delta}\right)^{*} \xi=-\xi$, namely

$$
\omega \in \Omega_{-}^{2}\left(C_{2}(M) ; F_{\rho}\right) \quad \text { and } \quad \xi \in \Omega_{-}^{2}\left(\Delta ; E_{\rho} \otimes E_{\rho}\right) .
$$

Furthermore, the cohomology class $[\xi] \in H_{-}^{2}\left(\Delta ; E_{\rho} \otimes E_{\rho}\right)$ is independent of the choice of $\xi$.

This proposition is proved in Section 3.

Now, we have the following 2-forms:

$$
\begin{aligned}
& q^{*} \pi_{1}^{*} \xi \in \Omega^{2}\left(C_{2}(M) ; q^{*}\left(E_{\rho}^{\otimes 2} \otimes \underline{\mathbb{R}}\right)\right), \\
& q^{*} \pi_{2}^{*} \xi \in \Omega^{2}\left(C_{2}(M) ; q^{*}\left(\underline{\mathbb{R}} \otimes E_{\rho}^{\otimes 2}\right)\right) .
\end{aligned}
$$

Then we obtain closed 6-forms

$$
\omega^{3} \in \Omega^{6}\left(C_{2}(M) ; F_{\rho}^{\otimes 3}\right) \quad \text { and }\left(q^{*} \pi_{1}^{*} \xi\right)\left(q^{*} \pi_{2}^{*} \xi\right) \omega \in \Omega^{6}\left(C_{2}(M) ; F_{\rho}^{\otimes 3}\right) .
$$

Since $F_{\rho}^{\otimes 3}=q^{*}\left(E_{\rho}^{\otimes 3} \otimes E_{\rho}^{\otimes 3}\right)$, the natural transformation $\operatorname{Tr}: E_{\rho}^{\otimes 3} \rightarrow \underline{\mathbb{R}}$ induces a natural transformation

$$
\operatorname{Tr}^{\otimes 2}: F_{\rho}^{\otimes 3} \longrightarrow(\underline{\mathbb{R}} \otimes \underline{\mathbb{R}}=) \underline{\mathbb{R}} .
$$

Therefore we get closed 6-forms

$$
\operatorname{Tr}^{\boxplus 2} \omega^{3}, \operatorname{Tr}^{\boxplus 2}\left(\left(q^{*} \pi_{1}^{*} \xi\right)\left(q^{*} \pi_{2}^{*} \xi\right) \omega\right) \in \Omega^{6}\left(C_{2}(M) ; \mathbb{R}\right) .
$$


Definition 2.2. We set

$$
\begin{gathered}
Z_{\Theta}(\omega)=\int_{C_{2}(M)} \operatorname{Tr}^{\bigotimes 2} \omega^{3}, Z_{O-O}(\omega, \xi)=\int_{C_{2}(M)} \operatorname{Tr}^{\bigotimes 2}\left(\left(q^{*} \pi_{1}^{*} \xi\right)\left(q^{*} \pi_{2}^{*} \xi\right) \omega\right), \\
Z_{1}(M, \rho)=Z_{\Theta}(\omega)-3 Z_{O-O}(\omega, \xi) .
\end{gathered}
$$

Theorem 2.3. $Z_{1}(M, \rho)$ is an invariant of $M, \rho$ (independent of the choices of $\omega$ and $\xi)$. Furthermore, $Z_{1}(M, \rho)$ is invariant under homotopy of the framing.

This theorem is proved in Section 4.

Remark 2.4. When we can take $\xi=0$, obviously $Z_{O-O}(\omega, \xi)=0$ and then $Z_{1}(M, \rho)$ coincides with the $\Theta$-invariant $I_{(\Theta, \text { tr }, \text { tr })}(M)$ of the framed 3-manifold $M$ given in Theorem 2.5 in [4].

\section{Proof of Proposition 2.1}

In the following commutative diagram, the top horizontal line is a part of the long exact sequence of the pair $\left(C_{2}(M), \partial C_{2}(M)\right)$ and the bottom line is that of $\left(M^{2}, \Delta\right)$. Thanks to the excision theorem, the right vertical homomorphism $q^{*}$ is an isomorphism:

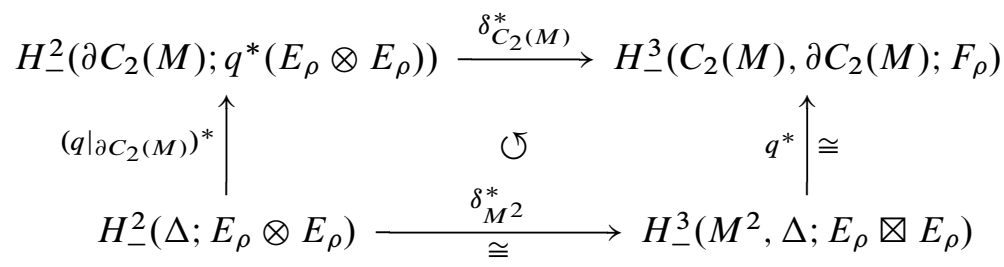

Since $H_{-}^{2}\left(M^{2} ; E_{\rho} \otimes E_{\rho}\right)=H_{-}^{3}\left(M^{2} ; E_{\rho} \otimes E_{\rho}\right)=0$, the homomorphism $\delta_{M^{2}}^{*}$ on the bottom line is an isomorphism. Set

$\Phi=\left(\delta_{M^{2}}^{*}\right)^{-1} \circ\left(q^{*}\right)^{-1} \circ \delta_{C_{2}(M)}^{*}: H_{-}^{2}\left(\partial C_{2}(M) ; q^{*}\left(E_{\rho} \otimes E_{\rho}\right)\right) \longrightarrow H_{-}^{2}\left(\Delta ; E_{\rho} \otimes E_{\rho}\right)$.

We take a closed 2-form $\xi \in \Omega_{-}^{2}\left(\Delta ; E_{\rho} \otimes E_{\rho}\right)$ such that

$$
\Phi\left(\left[p^{*} \omega_{S^{2}} \mathbf{1}\right]\right)=-[\xi] \in H_{-}^{2}\left(\Delta ; E_{\rho} \otimes E_{\rho}\right) .
$$

The above diagram implies that $\Phi\left(q^{*}[\xi]\right)=[\xi]$. Then $\Phi\left(p^{*} \omega_{S^{2}} \mathbf{1}+q^{*} \xi\right)=0$. Thus $\delta_{C_{2}(M)}^{*}\left(p^{*} \omega_{S^{2}} \mathbf{1}+q^{*} \xi\right)=0$. Therefore there exists a closed 2-form $\omega \in$ $\Omega_{-}^{2}\left(C_{2}(M) ; F_{\rho}\right)$ such that

$$
\left.\omega\right|_{\partial C_{2}(M)}=p^{*} \omega_{S^{2}} \mathbf{1}+q^{*} \xi .
$$


Conversely, if there exists a closed 2-form $\omega \in \Omega_{-}^{2}\left(C_{2}(M) ; F_{\rho}\right)$ such that $\left.\omega\right|_{\partial C_{2}(M)}=p^{*} \omega_{S^{2}} \mathbf{1}+q^{*} \xi$, then $\Phi\left(\left.\omega\right|_{\partial C_{2}(M)}\right)=0$ so that $[\xi]=-\Phi\left(\left[p^{*} \omega_{S^{2}} \mathbf{1}\right]\right)$.

\section{Proof of Theorem 2.3}

The proof is reduced to the following two propositions:

Proposition 4.1. Let $\omega, \omega^{\prime} \in \Omega_{-}^{2}\left(C_{2}(M) ; F_{\rho}\right)$ be closed 2-forms such that

$$
\left.\omega\right|_{\partial C_{2}(M)}=\left.\omega^{\prime}\right|_{\partial C_{2}(M)}=p^{*} \omega_{S^{2}} \mathbf{1}+q^{*} \xi .
$$

Then $Z_{\Theta}(\omega)=Z_{\Theta}\left(\omega^{\prime}\right)$ and $Z_{O-O}(\omega, \xi)=Z_{O-O}\left(\omega^{\prime}, \xi\right)$ hold.

Proposition 4.2. Let $\omega_{S^{2}, 0}, \omega_{S^{2}, 1} \in \Omega^{2}\left(S^{2} ; \mathbb{R}\right)$ be closed 2-forms satisfying

$$
\begin{gathered}
\int_{S^{2}} \omega_{S^{2}, 0}=\int_{S^{2}} \omega_{S^{2}, 1}=1, \\
T_{S^{2}}^{*} \omega_{S^{2}, 0}=-\omega_{S^{2}, 0} \quad \text { and } \quad T_{S^{2}}^{*} \omega_{S^{2}, 1}=-\omega_{S^{2}, 1} .
\end{gathered}
$$

Let $\left\{p_{t}: \Delta \times S^{2} \rightarrow S^{2}\right\}_{t \in[0,1]}$ be a homotopy such that $p_{0}=p$ and $\left.p_{t} \circ T\right|_{\partial C_{2}(M)}=$ $T_{S^{2}} \circ p_{t}$ for $t=0,1$. Let $\omega_{0}, \omega_{1} \in \Omega_{-}^{2}\left(C_{2}(M) ; F_{\rho}\right)$ and $\xi_{0}, \xi_{1} \in \Omega_{-}^{2}\left(\Delta ; E_{\rho} \otimes E_{\rho}\right)$ be closed 2-forms satisfying

$$
\left.\omega_{0}\right|_{\partial C_{2}(M)}=p_{0}^{*} \omega_{S^{2}, 0} \mathbf{1}+q^{*} \xi_{0},\left.\omega_{1}\right|_{\partial C_{2}(M)}=p_{1}^{*} \omega_{S^{2}, 1} \mathbf{1}+q^{*} \xi_{1} .
$$

Then

$$
Z_{\Theta}\left(\omega_{0}\right)-3 Z_{O-O}\left(\omega_{0}, \xi_{0}\right)=Z_{\Theta}\left(\omega_{1}\right)-3 Z_{O-O}\left(\omega_{1}, \xi_{1}\right)
$$

holds.

\subsection{Proof of Proposition 4.1}

Lemma 4.3. There exists a 1-form $\eta \in \Omega_{-}^{1}\left(M^{2} ; E_{\rho} \otimes E_{\rho}\right)$ such that

$$
\omega-\omega^{\prime}=d\left(q^{*} \eta\right) \text {. }
$$

Proof. In the following diagram, the top horizontal line is a part of the long exact sequence of the pair $\left(C_{2}(M), \partial C_{2}(M)\right)$ and the bottom line is that of $\left(M^{2}, \Delta\right)$. The left vertical homomorphism $q^{*}$ is an isomorphism because of the excision theorem:

$$
\begin{aligned}
& H_{-}^{2}\left(C_{2}(M), \partial C_{2}(M) ; F_{\rho}\right) \longrightarrow H_{-}^{2}\left(C_{2}(M) ; F_{\rho}\right) \\
& q^{*} \cong \quad \text { ज } q^{*} \uparrow \\
& H_{-}^{2}\left(M^{2}, \Delta ; E_{\rho} \otimes E_{\rho}\right) \longrightarrow H_{-}^{2}\left(M^{2} ; E_{\rho} \otimes E_{\rho}\right)
\end{aligned}
$$


The closed 2-form $\omega-\omega^{\prime}$ gives a cohomology class in $H_{-}^{2}\left(C_{2}(M), \partial C_{2}(M) ; F_{\rho}\right)$ and then $\left.\left(\left(q^{*}\right)^{-1}\left(\omega-\omega^{\prime}\right)\right)\right|_{M^{2}}$ gives a cohomology class in $H_{-}^{2}\left(M^{2} ; E_{\rho} \otimes E_{\rho}\right)$. Since $H_{-}^{2}\left(M^{2} ; E_{\rho} \otimes E_{\rho}\right)=0$, there exists a 1 -form $\eta \in \Omega_{-}^{1}\left(M^{2} ; E_{\rho} \otimes E_{\rho}\right)$ such that

$$
d \eta=\left.\left(\left(q^{*}\right)^{-1}\left(\omega-\omega^{\prime}\right)\right)\right|_{M^{2}}
$$

Thus we have $d\left(q^{*} \eta\right)=\omega-\omega^{\prime}$.

Thanks to Lemma 4.3 and Stokes's theorem,

$$
\begin{aligned}
Z_{\Theta}(\omega)-Z_{\Theta}\left(\omega^{\prime}\right) & =\int_{C_{2}(M)} \operatorname{Tr}^{\bigotimes 2}\left(\left(\omega-\omega^{\prime}\right)\left(\omega^{2}+\omega \omega^{\prime}+\omega^{\prime 2}\right)\right) \\
& =\int_{C_{2}(M)} \operatorname{Tr}^{\bigotimes 2}\left(d\left(q^{*} \eta\right)\left(\omega^{2}+\omega \omega^{\prime}+\omega^{\prime 2}\right)\right) \\
& =\int_{\partial C_{2}(M)} \operatorname{Tr}^{\bigotimes 2}\left(\left.\left.\left(q^{*} \eta\right)\right|_{\partial C_{2}(M)}\left(\omega^{2}+\omega \omega^{\prime}+\omega^{\prime 2}\right)\right|_{\partial C_{2}(M)}\right) \\
& =3 \int_{\partial C_{2}(M)} \operatorname{Tr}^{\boxplus 2}\left(\left.\left(q^{*} \eta\right)\right|_{\partial C_{2}(M)}\left(p^{*} \omega_{S^{2}} \mathbf{1}+q^{*} \xi\right)^{2}\right) \\
& =6 \int_{\Delta \times S^{2}} \operatorname{Tr}^{\boxplus 2}\left(q^{*}\left(\left.\eta\right|_{\Delta}\right) p^{*} \omega_{S^{2}} \mathbf{1} q^{*} \xi\right) \\
& =6 \int_{\Delta} \operatorname{Tr}^{\boxplus 2}\left(\left.\eta\right|_{\Delta \xi} \mathbf{1}\right) .
\end{aligned}
$$

To simplify the notation, we set $\bar{\eta}=\left.\eta\right|_{\Delta}$.

Let $l: E_{\rho} \otimes E_{\rho} \rightarrow E_{\rho}$ be a natural transformation induced from the Lie bracket [,]: $\mathfrak{g} \otimes \mathfrak{g} \rightarrow \mathfrak{g}$. We have $l_{*}(\bar{\eta}) \in \Omega^{1}\left(\Delta ; E_{\rho}\right), l_{*}(\xi) \in \Omega^{2}\left(\Delta ; E_{\rho}\right)$. Let $I: E_{\rho} \otimes E_{\rho} \rightarrow \underline{\mathbb{R}}$ be a natural transformation induced from the inner product of $\mathfrak{g}$. Then $I_{*}\left(l_{*}(\bar{\eta}) l_{*}(\xi)\right)$ is a 3 -form in $\Omega^{3}(\Delta ; \mathbb{R})$.

Lemma 4.4. $\operatorname{Tr}^{\otimes 2}(\bar{\eta} \xi \mathbf{1})=\frac{1}{2} I_{*}\left(l_{*}(\bar{\eta}) l_{*}(\xi)\right)$.

Proof. Since $\left.\left.T_{0}\right|_{\Delta}=\mathrm{id}, \Omega_{-}^{*}(\Delta ; E \otimes E)\right)=\Omega^{*}\left(\Delta ;(E \otimes E)_{-}\right)$. Then we only need to check the claim on $\mathfrak{g}^{\otimes 3} \otimes \mathfrak{g}^{\otimes 3}$. Let $e_{1}, \ldots, e_{\operatorname{dim} \mathfrak{g}} \in \mathfrak{g}$ be an orthonormal basis of $\mathfrak{g}$. Then $\left\{e_{i} \otimes e_{j}-e_{j} \otimes e_{i}: i<j\right\}$ is a basis of $(\mathfrak{g} \otimes \mathfrak{g})^{-}$. It is enough to 
show the claim for this basis:

$$
\begin{aligned}
\operatorname{Tr}^{\otimes 2} & \left(\left(e_{i} \otimes e_{j}-e_{j} \otimes e_{i}\right) \otimes\left(e_{k} \otimes e_{l}-e_{l} \otimes e_{k}\right) \otimes\left(\sum_{n} e_{n} \otimes e_{n}\right)\right) \\
& =2\left(\left\langle\left[e_{i}, e_{k}\right],\left[e_{j}, e_{l}\right]\right\rangle-\left\langle\left[e_{i}, e_{l}\right],\left[e_{j}, e_{k}\right]\right\rangle\right) \\
& =2\left(\left\langle e_{i},\left[e_{k},\left[e_{j}, e_{l}\right]\right]\right\rangle+\left\langle e_{i},\left[e_{l},\left[e_{k}, e_{j}\right]\right]\right\rangle\right) \\
& =2\left(\left(-\left\langle e_{i},\left[e_{j},\left[e_{l}, e_{k}\right]\right]\right\rangle-\left\langle e_{i},\left[e_{l},\left[e_{k}, e_{j}\right]\right]\right\rangle\right)+\left\langle e_{i},\left[e_{l},\left[e_{k}, e_{j}\right]\right]\right\rangle\right) \\
& =2\left\langle e_{i},\left[e_{j},\left[e_{k}, e_{l}\right]\right]\right\rangle \\
& =2\left\langle\left[e_{i}, e_{j}\right],\left[e_{k}, e_{l}\right]\right\rangle \\
& =\frac{1}{2}\left\langle 2\left[e_{i}, e_{j}\right], 2\left[e_{k}, e_{l}\right]\right\rangle \\
& =\frac{1}{2}\left\langle l\left(e_{i} \otimes e_{j}-e_{j} \otimes e_{i}\right) l\left(e_{k} \otimes e_{l}-e_{l} \otimes e_{k}\right)\right\rangle .
\end{aligned}
$$

Corollary 4.5. $\int_{\Delta} \operatorname{Tr}^{\boxplus 2}(\bar{\eta} \xi \mathbf{1})=0$.

Proof. Thanks to the above lemma,

$$
\int_{\Delta} \operatorname{Tr}^{\otimes 2}(\bar{\eta} \xi \mathbf{1})=\frac{1}{2} \int_{\Delta} I_{*}\left(l_{*}(\bar{\eta}) l_{*}(\xi)\right) .
$$

Since $E_{\rho}$ is acyclic, $\left[l_{*}(\xi)\right]=0 \in H^{2}\left(\Delta ; E_{\rho}\right)=0$. Thus there exists a 1 -form $\zeta \in \Omega^{1}\left(\Delta ; E_{\rho}\right)$ such that $d \zeta=l_{*}(\xi)$. Therefore

$$
\begin{aligned}
\int_{\Delta} I_{*}\left(l_{*}(\bar{\eta}) l_{*}(\xi)\right) & =\int_{\Delta} I_{*}\left(l_{*}(\bar{\eta}) d \zeta\right) \\
& =\int_{\Delta} I_{*}\left(d l_{*}(\bar{\eta}) \zeta\right)-\int_{\Delta} d I_{*}\left(l_{*}(\bar{\eta}) \zeta\right) .
\end{aligned}
$$

The first term of the last line is vanishing because $d l_{*}(\bar{\eta})=l_{*}\left(\left.d \eta\right|_{\Delta}\right)$ and $d \eta=0$ on $\Delta$. Thus we have

$$
\int_{\Delta} I_{*}\left(l_{*}(\bar{\eta}) l_{*}(\xi)\right)=-\int_{\Delta} d I_{*}\left(l_{*}(\bar{\eta}) \zeta\right)=0 .
$$

Thanks to the above lemma, we have

$$
Z_{\Theta}(\omega)-Z_{\Theta}\left(\omega^{\prime}\right)=0
$$


Similarly,

$$
\begin{aligned}
Z_{O-O}(\omega, \xi)-Z_{O-O}\left(\omega^{\prime}, \xi\right) & =\int_{C_{2}(M)} \operatorname{Tr}^{\boxplus 2}\left(\left(q^{*} \pi_{1}^{*} \xi\right)\left(q^{*} \pi_{2}^{*} \xi\right)\left(\omega-\omega^{\prime}\right)\right) \\
& =\int_{C_{2}(M)} \operatorname{Tr}^{\boxplus 2}\left(\left(q^{*} \pi_{1}^{*} \xi\right)\left(q^{*} \pi_{2}^{*} \xi\right) d q^{*} \eta\right) \\
& =\int_{\partial C_{2}(M)} \operatorname{Tr}^{\bigotimes 2}\left(q^{*}\left(\left(\left.\pi_{1}\right|_{\Delta}\right)^{*} \xi\left(\left.\pi_{2}\right|_{\Delta}\right)^{*} \xi \bar{\eta}\right)\right) .
\end{aligned}
$$

Since $\left(\left.\pi_{1}\right|_{\Delta}\right)^{*} \xi\left(\left.\pi_{2}\right|_{\Delta}\right)^{*} \xi \bar{\eta}$ is a 5-form on the 3-dimensional manifold $\Delta$, the last term is vanishing. This completes the proof of Proposition 4.1.

4.2. Proof of Proposition 4.2. Since $\left[\omega_{S^{2}, 0}\right]=\left[\omega_{S^{2}, 1}\right] \in H^{2}\left(S^{2} ; \mathbb{R}\right)$, there exists a closed 2-form $\widetilde{\omega_{S_{2}}} \in \Omega^{2}\left([0,1] \times S^{2} ; \mathrm{R}\right)$ such that $\left.\widetilde{\omega_{S^{2}}}\right|_{\{t\} \times S^{2}}=\omega_{S^{2}, t}$ for $t=0,1$.

Since $\left[\xi_{0}\right]=\left[\xi_{1}\right]($ Proposition 2.1), there exists a closed 1-form

$$
\tilde{\xi} \in \Omega^{1}\left([0,1] \times \Delta, \pi_{\Delta}^{*}\left(E_{\rho} \otimes E_{\rho}\right)\right)
$$

such that $\left.\tilde{\xi}\right|_{\{0\} \times \Delta}=\xi_{0}$ and $\left.\tilde{\xi}\right|_{\{1\} \times \Delta}=\xi_{1}$. Here $\pi_{\Delta}:[0,1] \times \Delta \rightarrow \Delta$ is the projection.

Let $\pi_{C_{2}(M)}:[0,1] \times C_{2}(M) \rightarrow C_{2}(M)$ be the projection. Let

$$
\tilde{q}=\operatorname{id}_{[0,1]} \times q:[0,1] \times C_{2}(M) \longrightarrow[0,1] \times M^{2}
$$

and we also denote the restriction map

$$
\left.\tilde{q}\right|_{[0,1] \times \partial C_{2}(M):[0,1] \times \partial C_{2}(M) \longrightarrow[0,1] \times \Delta}
$$

as $\tilde{q}$. By a similar argument as in Proposition 2.1, we can take a closed 2-form

$$
\tilde{\omega} \in \Omega^{2}\left([0,1] \times C_{2}(M), \pi_{C_{2}(M)}^{*} F_{\rho}\right)
$$

such that

$$
\left.\tilde{\omega}\right|_{[0,1] \times \partial C_{2}(M)}=\tilde{p}^{*} \widetilde{\omega_{S^{2}}} \mathbf{1}+\tilde{q}^{*} \tilde{\xi}
$$

Here

$$
\tilde{p}=\left\{p_{t}\right\}_{t}:\left([0,1] \times \partial C_{2}(M)=\right)[0,1] \times \Delta \times S^{2} \longrightarrow S^{2}
$$

is the homotopy between $p_{0}$ and $p_{1}$. 
Thanks to Proposition 4.1, both $Z_{\Theta}(\omega)$ and $Z_{O-O}(\omega, \xi)$ depend only on $\left.\omega\right|_{\Delta \times S^{2}}$ and $\xi$. Thus we have

$$
\begin{aligned}
Z_{\Theta}\left(\omega_{0}\right) & =Z_{\Theta}\left(\left.\tilde{\omega}\right|_{\{0\} \times C_{2}(M)}\right), \\
Z_{\Theta}\left(\omega_{1}\right) & =Z_{\Theta}\left(\left.\tilde{\omega}\right|_{\{1\} \times C_{2}(M)}\right), \\
Z_{O-O}\left(\omega_{0}, \xi_{0}\right) & =Z_{O-O}\left(\left.\tilde{\omega}\right|_{\{0\} \times C_{2}(M)}, \xi_{0}\right), \\
Z_{O-O}\left(\omega_{1}, \xi_{1}\right) & =Z_{O-O}\left(\left.\tilde{\omega}\right|_{\{1\} \times C_{2}(M)}, \xi_{1}\right) .
\end{aligned}
$$

We note that, with our orientation convention,

$$
\partial\left([0,1] \times C_{2}(M)\right)=\{1\} \times C_{2}(M)-\{0\} \times C_{2}(M)-[0,1] \times \partial C_{2}(M) .
$$

Therefore, by using Stokes' theorem,

$$
\begin{aligned}
0 & =\int_{[0,1] \times C_{2}(M)} d \operatorname{Tr}^{\bigotimes 2} \tilde{\omega}^{3} \\
= & \int_{\{1\} \times C_{2}(M)} \operatorname{Tr}^{\bigotimes 2}\left(\left.\tilde{\omega}\right|_{\{1\} \times C_{2}(M)} ^{3}\right)-\int_{\{0\} \times C_{2}(M)} \operatorname{Tr}^{\bigotimes 2}\left(\left.\tilde{\omega}\right|_{\{0\} \times C_{2}(M)} ^{3}\right. \\
& \quad-\int_{[0,1] \times \partial C_{2}(M)} \operatorname{Tr}^{\bigotimes 2}\left(\left.\tilde{\omega}\right|_{[0,1] \times \partial C_{2}(M)} ^{3}\right) \\
= & Z_{\Theta}\left(\left.\tilde{\omega}\right|_{\{1\} \times C_{2}(M)}\right)-Z_{\Theta}\left(\left.\tilde{\omega}\right|_{\{0\} \times C_{2}(M)}\right)-\int_{[0,1] \times \partial C_{2}(M)} \operatorname{Tr}^{\boxplus 2}\left(\tilde{p}^{*} \widetilde{\omega_{S^{2}}} \mathbf{1}+\tilde{q}^{*} \tilde{\xi}\right)^{3} \\
= & Z_{\Theta}\left(\omega_{1}\right)-Z_{\Theta}\left(\omega_{0}\right)-\int_{[0,1] \times \partial C_{2}(M)} \operatorname{Tr}^{\bigotimes 2}\left(3 \tilde{p}^{*} \widetilde{\omega_{S^{2}}} \mathbf{1} \tilde{q}^{*} \tilde{\xi}^{2}\right)
\end{aligned}
$$

We denote

$$
\tilde{\pi}_{i}=\operatorname{id}_{[0,1]} \times \pi_{i}:[0,1] \times M^{2} \longrightarrow[0,1] \times M \quad \text { for } i=1,2 .
$$

We have

$$
\begin{aligned}
& 0=\int d \operatorname{Tr}^{\bigotimes 2}\left(\left(\tilde{q}^{*} \tilde{\pi}_{1}{ }^{*} \tilde{\xi}\right)\left(\tilde{q}^{*} \tilde{\pi}_{2}{ }^{*} \tilde{\xi}\right) \tilde{\omega}\right) \\
& {[0,1] \times C_{2}(M)} \\
& =Z_{O \smile O}\left(\omega_{1}, \xi_{1}\right)-Z_{O \smile O}\left(\omega_{0}, \xi_{0}\right) \\
& -\int \operatorname{Tr}^{\boxplus 2}\left(\left.\left(\tilde{q}^{*}\left(\left.\widetilde{\pi}_{1}\right|_{[0,1] \times \Delta}\right)^{*} \tilde{\xi}\left(\left.\widetilde{\pi}_{2}\right|_{[0,1] \times \Delta}\right)^{*} \tilde{\xi}\right) \tilde{\omega}\right|_{[0,1] \times \partial C_{2}(M)}\right) \text {. } \\
& {[0,1] \times \partial C_{2}(M)}
\end{aligned}
$$


Here,

$$
\left.\widetilde{\pi_{1}}\right|_{[0,1] \times \Delta}=\left.\widetilde{\pi_{2}}\right|_{[0,1] \times \Delta}:[0,1] \times \Delta \longrightarrow M
$$

Thus

$$
\left(\left.\widetilde{\pi}_{1}\right|_{[0,1] \times \Delta}\right)^{*} \tilde{\xi}\left(\left.\widetilde{\pi_{2}}\right|_{[0,1] \times \Delta}\right)^{*} \tilde{\xi}=\tilde{\xi}^{2}
$$

under the identification $\Delta=M$. We have

$$
Z_{O-O}\left(\omega_{1}, \xi_{1}\right)-Z_{O-O}\left(\omega_{0}, \xi_{0}\right)=\int_{[0,1] \times \partial C_{2}(M)} \operatorname{Tr}^{\boxplus 2}\left(\tilde{p}^{*} \widetilde{\omega_{S^{2}}} 1 \tilde{q}^{*} \tilde{\xi}^{2}\right)
$$

Then,

$$
Z_{\Theta}\left(\omega_{1}\right)-Z_{\Theta}\left(\omega_{0}\right)=3\left(Z_{O-O}\left(\omega_{1}, \xi_{1}\right)-Z_{O-O}\left(\omega_{0}, \xi_{0}\right)\right) .
$$

This completes the proof of Proposition 4.2.

\section{A framing correction}

In this section, we introduce a correction term for framings to give an invariant of closed 3-manifolds equipped with acyclic representations. Let $M$ be a closed oriented 3-manifold (without framings). Recall that $\partial C_{2}(M)$ is identified with the unit sphere bundle $S T M$ (see Section 2.1). Take a framing $f: T M \rightarrow M \times \mathbb{R}^{3}$ of $M$. Then $(M, f)$ is a framed 3-manifold. Let $p:\left(\partial C_{2}(M)=\right) S T M \rightarrow S^{2}$ be the projection defined by the framing $f$. Let $\delta(f) \in \mathbb{Z}$ be the signature defect (or Hirzebruch defect, see [1] or [5] for the details) of a framing $f$. For the convenience of the reader, we give a short review of the construction of $\delta(f)$ in the next section. Let $\rho: \pi_{1}(M) \rightarrow G$ be an acyclic representation as in Section 2.1.

Theorem 5.1. $Z_{1}((M, f), \rho)-\operatorname{Tr}^{\bigotimes 2}\left(\mathbf{1}^{\otimes 3}\right) \delta(f)$ is a topological invariant of $M, \rho$.

5.1. The signature defect $\delta(p)$. Let $W$ be a compact 4-manifold such that $\partial W=M$ and its Euler characteristic is zero. Then there exists an $\mathbb{R}^{3}$ subbundle $T^{v} W$ of $T W$ satisfying $\left.T^{v} W\right|_{M}=T M$. Let $S T^{v} W \rightarrow W$ be the unit sphere bundle of $T^{v} W \rightarrow W$. Thus $S T^{v} W$ is a 6-dimensional manifold with $\partial S T^{v} W=S T M$. We denote by $F_{W} \rightarrow S T^{v} W$ the tangent bundle along the fiber of the $S^{2}$ bundle $\pi: S T^{v} W \rightarrow W$.

Take a closed 2-form $\alpha_{W} \in \Omega^{2}\left(S T^{v} W ; \mathbb{R}\right)$ such that $\left.\alpha_{W}\right|_{S T M}=p^{*} \omega_{S^{2}}$ and $\left[\alpha_{W}\right]=e\left(F_{W}\right) / 2 \in H^{2}\left(S T^{v} W ; \mathbb{R}\right)$, where $e\left(F_{W}\right)$ is the Euler class of $F_{W} \rightarrow S T^{v} W$. 
Lemma 5.2. When $\partial W=M=\emptyset$,

$$
\int_{S T^{v} W} \alpha_{W}^{3}=\frac{3}{4} \operatorname{Sign} W .
$$

Here Sign $W$ is the signature of $W$.

Proof. We give an outline of the proof. See Appendix of [8] or Proposition 2.45 of [7], for the details of the proof.

Since $W$ is closed, $\int_{S T^{v} W} \alpha_{W}^{3}=\int_{S T^{v} W}\left(\frac{1}{2} e\left(F_{W}\right)\right)^{3}$. We denote by $p_{1}\left(F_{W}\right) \in$ $H^{4}\left(S T^{v} W ; \mathbb{R}\right)$ the first Pontrjagin class of the bundle $F_{W}$. We remark that $\underline{\mathbb{R}} \oplus F_{W}=\pi^{*} T^{v} W$ and $\underline{\mathbb{R}} \oplus T^{v} W=T W$. Here $\underline{\mathbb{R}}$ is the trivial $\mathbb{R}$ bundle over an appropriate manifold. Therefore,

$$
\begin{aligned}
\int_{S T^{v} W} \alpha_{W}^{3} & =\frac{1}{8} \int_{S T^{v} W} e\left(F_{W}\right)^{3} \\
& =\frac{1}{8} \int_{S T^{v} W} e\left(F_{W}\right) p_{1}\left(F_{W}\right) \\
& =\frac{1}{8} \int_{S T^{v} W} e\left(F_{W}\right) \pi^{*} p_{1}\left(T^{v} W\right) \\
& =\frac{1}{4} \int_{W} p_{1}(T W) \\
& =\frac{3}{4} \operatorname{Sign} W .
\end{aligned}
$$

Thanks to the Novikov additivity for the signature, the following corollary holds.

Corollary 5.3. The signature defect $\delta(f)$, defined as

$$
\delta(f)=\int_{S T^{v} W} \alpha_{W}^{3}-\frac{3}{4} \operatorname{Sign} W,
$$

is independent of the choices of $W$ and $\alpha_{W}$.

5.2. Proof of Theorem 5.1. Let $f_{0}, f_{1}: T M \rightarrow M \times \mathbb{R}^{3}$ be framings and let $p_{0}, p_{1}: \partial C_{2}(M) \rightarrow S^{2}$ be the projections given by framings $f_{0}, f_{1}$ respectively. Since $\left[p_{0}^{*} \omega_{S^{2}}\right]$ and $\left[p_{1}^{*} \omega_{S^{2}}\right]$ are in $H_{-}^{2}\left(\Delta \times S^{2} ; \mathbb{R}\right)=H^{2}\left(S^{2} ; \mathbb{R}\right)=\mathbb{R},\left[p_{0}^{*} \omega_{S^{2}}\right]=$ $\left[p_{1}^{*} \omega_{S^{2}}\right]$. Thus there exists a closed 2-form

$$
\tilde{\omega}_{\partial} \in \Omega_{-}^{2}\left([0,1] \times \partial C_{2}(M) ; \mathbb{R}\right)
$$


such that

$$
\left.\tilde{\omega}_{\partial}\right|_{\{0\} \times \partial C_{2}(M)}=p_{0}^{*} \omega_{S^{2}} \text { and }\left.\quad \tilde{\omega}_{\partial}\right|_{\{1\} \times \partial C_{2}(M)}=p_{1}^{*} \omega_{S^{2}} .
$$

We recall that $(-\xi) \in \Omega_{-}^{2}\left(\Delta ; E_{\rho} \otimes E_{\rho}\right)$ is a closed 2-form representing

$$
\Phi\left(\left[p^{*} \omega_{S^{2}} \mathbf{1}\right]\right)=\Phi \circ c_{*}\left(\left[p^{*} \omega_{S^{2}}\right]\right)
$$

when we take a projection $p: \partial C_{2}(M) \rightarrow S^{2}$ given by a framing $f$. The homomorphism $\Phi \circ c_{*}$ is independent from the choice of a framing. Then we can use the same $\xi \in \Omega_{-}^{2}\left(\Delta ; E_{\rho} \otimes E_{\rho}\right)$ for any framing.

By a similar argument as in proof of Proposition 2.1, we can take a closed 2-form

such that

$$
\tilde{\omega} \in \Omega^{2}\left([0,1] \times C_{2}(M) ; \pi_{C_{2}(M)}^{*} F_{\rho}\right)
$$

$$
\left.\tilde{\omega}\right|_{[0,1] \times \partial C_{2}(M)}=\tilde{\omega}_{\partial} \mathbf{1}+Q^{*} \xi .
$$

Here, $\pi_{C_{2}(M)}:[0,1] \times C_{2}(M) \rightarrow C_{2}(M)$ and $Q:[0,1] \times \partial C_{2}(M) \rightarrow \Delta$ are the projections. We denote by

$$
\begin{aligned}
& \omega_{0}=\left.\tilde{\omega}\right|_{\{0\} \times C_{2}(M)}, \\
& \omega_{1}=\left.\tilde{\omega}\right|_{\{1\} \times C_{2}(M)} .
\end{aligned}
$$

Then,

$$
\begin{aligned}
& Z_{1}\left(\left(M, f_{0}\right), \rho\right)=Z_{\Theta}\left(\omega_{0}\right)-3 Z_{O-O}\left(\omega_{0}, \xi\right), \\
& Z_{1}\left(\left(M, f_{1}\right), \rho\right)=Z_{\Theta}\left(\omega_{1}\right)-3 Z_{O-O}\left(\omega_{1}, \xi\right) .
\end{aligned}
$$

Thanks to Stokes' theorem,

$$
\begin{aligned}
& 0=\int d \operatorname{Tr}^{\otimes 2}\left(\tilde{\omega}^{3}\right) \\
& {[0,1] \times C_{2}(M)} \\
& =Z_{\Theta}\left(\omega_{1}\right)-Z_{\Theta}\left(\omega_{0}\right)-\int \operatorname{Tr}^{\bigotimes 2}\left(\left.\tilde{\omega}\right|_{[0,1] \times \partial C_{2}(M)} ^{3}\right) \\
& {[0,1] \times \partial C_{2}(M)} \\
& =Z_{\Theta}\left(\omega_{1}\right)-Z_{\Theta}\left(\omega_{0}\right)-\int_{[0,1] \times \partial C_{2}(M)} \operatorname{Tr}^{\bigotimes 2}\left(\tilde{\omega}_{\partial}^{3} \mathbf{1}^{\otimes 3}\right)-\int_{[0,1] \times \partial C_{2}(M)} 3 \operatorname{Tr}^{\bigotimes 2}\left(\tilde{\omega}_{\partial}^{2} \mathbf{1}^{\otimes 2} Q^{*} \xi\right) \\
& =Z_{\Theta}\left(\omega_{1}\right)-Z_{\Theta}\left(\omega_{0}\right)-\int \tilde{\omega}_{\partial}^{3} \operatorname{Tr}^{\boxplus 2}\left(\mathbf{1}^{\otimes 3}\right)-\int 3 \operatorname{Tr}^{\boxplus 2}\left(\tilde{\omega}_{\partial}^{2} 1^{\otimes 2} Q^{*} \xi\right) \\
& {[0,1] \times \partial C_{2}(M) \quad[0,1] \times \partial C_{2}(M)} \\
& =Z_{\Theta}\left(\omega_{1}\right)-Z_{\Theta}\left(\omega_{0}\right)-\operatorname{Tr}^{\bigotimes 2}\left(\mathbf{1}^{\otimes 3}\right) \int_{\tilde{\omega}_{\partial}^{3}}-\int_{\operatorname{Cr}} 3 \operatorname{Tr}^{\bigotimes 2}\left(\tilde{\omega}_{\partial}^{2} \mathbf{1}^{\otimes 2} Q^{*} \xi\right) .
\end{aligned}
$$


We denote $\bar{\pi}_{i}:[0,1] \times M^{2} \rightarrow M,\left(t, x_{1}, x_{2}\right) \mapsto x_{i}$ for $i=1,2$. We have,

$$
\begin{aligned}
& \int_{[0,1] \times C_{2}(M)} d \operatorname{Tr}^{\boxplus 2}\left(\left(\tilde{q}^{*} \bar{\pi}_{1}^{*} \xi\right)\left(\tilde{q}^{*} \bar{\pi}_{2}^{*} \xi\right) \tilde{\omega}\right) \\
& =Z_{O \frown O}\left(\omega_{1}, \xi\right)-Z_{O \smile O}\left(\omega_{0}, \xi\right) \\
& -\int \operatorname{Tr}^{\boxplus 2}\left(\left(\tilde{q}^{*}\left(\left.\bar{\pi}_{1}\right|_{[0,1] \times \Delta}\right)^{*} \xi\left(\left.\bar{\pi}_{2}\right|_{[0,1] \times \Delta}\right)^{*} \xi\right) \tilde{\omega}_{\partial} \mathbf{1}\right) \\
& {[0,1] \times \partial C_{2}(M)} \\
& =Z_{O-O}\left(\omega_{1}, \xi\right)-Z_{O-O}\left(\omega_{0}, \xi\right)-\int_{[0,1] \times \partial C_{2}(M)} \operatorname{Tr}^{\otimes 2}\left(Q^{*} \xi^{2} \tilde{\omega}_{\partial} \mathbf{1}\right) \\
& =Z_{O-O}\left(\omega_{1}, \xi\right)-Z_{O-O}\left(\omega_{0}, \xi\right) .
\end{aligned}
$$

Thus we have

$$
\begin{aligned}
& Z_{1}\left(\left(M, f_{0}\right), \rho\right)-Z_{1}\left(\left(M, f_{1}\right), \rho\right) \\
& =\operatorname{Tr}^{\otimes 2}\left(\mathbf{1}^{\otimes 3}\right) \int_{[0,1] \times \partial C_{2}(M)} \tilde{\omega}_{\partial}^{3}+\int_{[0,1] \times \partial C_{2}(M)} 3 \operatorname{Tr}^{\bigotimes 2}\left(\tilde{\omega}_{\partial}^{2} \mathbf{1}^{\otimes 2} Q^{*} \xi\right) .
\end{aligned}
$$

Lemma 5.4. $\operatorname{Tr}^{\otimes 2}\left(\tilde{\omega}_{\partial} \mathbf{1}^{\otimes 2} Q^{*} \xi\right)=0$.

Proof. Let

$$
T_{E}: E_{\rho} \otimes E_{\rho} \longrightarrow E_{\rho} \otimes E_{\rho}
$$

be the involution induced by

$$
\mathfrak{g} \otimes \mathfrak{g} \longrightarrow \mathfrak{g} \otimes \mathfrak{g}, \quad x \otimes y \longmapsto y \otimes x .
$$

Clearly,

$$
\operatorname{Tr}^{\otimes 2} \circ T_{E}^{\otimes 3}=\operatorname{Tr}^{\otimes 2}: E^{\otimes 3} \otimes E^{\otimes 3} \longrightarrow \underline{\mathbb{R}} .
$$

Since $T_{E}(\mathbf{1})=\mathbf{1}$ and $T_{E}^{*}=\left(\left.T_{0}\right|_{\Delta}\right)^{*}$ on $\Omega^{1}\left(\Delta ; E_{\rho} \otimes E_{\rho}\right)$,

$$
\operatorname{Tr}^{\bigotimes 2}\left(\tilde{\omega}_{\partial} \mathbf{1}^{\otimes 2} Q^{*} \xi\right)=\operatorname{Tr}^{\bigotimes 2}\left(T_{E}^{\otimes 3}\left(\tilde{\omega}_{\partial} \mathbf{1}^{\otimes 2} Q^{*} \xi\right)\right)=-\operatorname{Tr}^{\bigotimes 2}\left(\tilde{\omega}_{\partial} \mathbf{1}^{\otimes 2} Q^{*} \xi\right) .
$$

Thus $\operatorname{Tr}^{\bigotimes 2}\left(\tilde{\omega}_{\partial} \mathbf{1}^{\otimes 2} Q^{*} \xi\right)=0$.

Lemma 5.5. We have

$$
\delta\left(f_{1}\right)-\delta\left(f_{0}\right)=\int \tilde{\omega}_{\partial}^{3} .
$$


Proof. We take a compact 4-manifold $W$ with $\partial W=M$ and its Euler characteristic is zero. Take a collar neighborhood $[0,1] \times \partial M$ of $M=\partial W$ in $W$ such that $\{1\} \times M=\partial W$. Set

$$
W_{0}=W \backslash([0,1] \times M) .
$$

We can take $T^{v} W$ as $\left.T^{v} W\right|_{[0,1] \times M}=[0,1] \times T M$. Thus $\left.S T^{v} W\right|_{[0,1] \times M}$ is identified with $[0,1] \times \partial C_{2}(M)$. Take a closed 2-form $\alpha_{W} \in \Omega^{2}\left(S T^{v} W ; \mathbb{R}\right)$ satisfying $\left.\alpha_{W}\right|_{[0,1] \times S T M}=\tilde{\omega}_{\partial}$ and $\left[\alpha_{W}\right]=\frac{1}{2} e\left(F_{W}\right)$. Then we have

$$
\begin{aligned}
\delta\left(f_{1}\right)-\delta\left(f_{0}\right) & =\left(\int_{S T^{v} W} \alpha_{W}^{3}-\frac{3}{4} \operatorname{Sign} W\right)-\left(\int_{S T^{v} W_{0}}\left(\alpha_{W} \mid S T^{v} W_{0}\right)^{3}-\frac{3}{4} \operatorname{Sign} W_{0}\right) \\
& =\int_{[0,1] \times S T M}\left(\alpha_{W} \mid[0,1] \times S T M\right)^{3} \\
& =\int \tilde{\omega}_{\partial}^{3} . \\
{[0,1] \times \partial C_{2}(M) } &
\end{aligned}
$$

By the above two lemmas,

$$
Z_{1}\left(\left(M, f_{0}\right), \rho\right)-\operatorname{Tr}^{\otimes 2}\left(\mathbf{1}^{\otimes 3}\right) \delta\left(f_{0}\right)=Z_{1}\left(\left(M, f_{1}\right), \rho\right)-\operatorname{Tr}^{\bigotimes 2}\left(\mathbf{1}^{\otimes 3}\right) \delta\left(f_{1}\right) .
$$

Namely, $Z_{1}((M, f), \rho)-\operatorname{Tr}^{\bigotimes 2}\left(\mathbf{1}^{\otimes 3}\right) \delta(f)$ is independent of the choice of a framing $f$.

\section{References}

[1] M. Atiyah, On framings of 3-manifolds. Topology 29 (1990), no. 1, 1-7. MR 1046621 Zbl 0716.57011

[2] S. Axelrod and I. M. Singer, Chern-Simons perturbation theory. In S. Catto and A. Rocha (eds.), Proceedings of the XXth International Conference on Differential Geometric Methods in Theoretical Physics. Vol. 1, 2. Held at the Bernard M. Baruch College of the City University of New York, June 3-7, 1991. World Scientific Publishing Co., River Edge, N.J., 1992, 3-45. MR 1225107 Zbl 0813.53051

[3] R. Bott and A. S. Cattaneo, Integral invariants of 3-manifolds. J. Differential Geom. 48 (1998), no. 1, 91-133. MR 1622602 Zbl 0953.57008

[4] R. Bott and A. S. Cattaneo, Integral invariants of 3-manifolds. II. J. Differential Geom. 53 (1999), no. 1, 1-13. MR 1776090 Zbl 1036.57500

[5] R. Kirby and P. Melvin, Canonical framings for 3-manifolds. Turkish J. Math. 23 (1999), no. 1, 89-115. MR 1701641 Zbl 0947.57020 
[6] M. Kontsevich, Feynman diagrams and low-dimensional topology. In A. Joseph, F. Mignot, F. Murat, B. Prum, and R. Rentschler (eds.), First European Congress of Mathematics. Vol. II. Invited lectures. Part 2. Proceedings of the congress held at the Sorbonne and Panthéon-Sorbonne Universities, Paris, July 6-10, 1992. Progress in Mathematics, 120. Birkhäuser Verlag, Basel, 1994, 97-121. MR 1341841 Zbl 0872.57001

[7] C. Lescop, Kontsevich-Kuperberg-Thurston construction of a configuration-space invariant for rational homology 3-spheres. Preprint, 2004.

arXiv:math/0411088 [math.GT]

[8] T. Shimizu, An invariant of rational homology 3-spheres via vector fields. Algebr. Geom. Topol. 16 (2016), no. 6, 3073-3101. MR 3584254 Zbl 1357.57040

[9] T. Shimizu, Morse homotopy for the $S U(2)$-Chern-Simons perturbation theory. Preprint, 2016. RIMS preprint 1857

Received May 31, 2019

Alberto S. Cattaneo, Institute of Mathematics, University of Zurich, 190,

Winterthurerstrasse, 8057 Zürich, Switzerland

e-mail: cattaneo@math.uzh.ch

Tatsuro Shimizu, Research Institute for Mathematical Sciences, Kyoto University, Kyoto 606-8502, Japan

e-mail: shimizu@kurims.kyoto-u.ac.jp 